On Babinet's principle and a diffraction-interferometric technique to determine the diameter of cylindrical wires

This content has been downloaded from IOPscience. Please scroll down to see the full text. 2001 Metrologia 38125

(http://iopscience.iop.org/0026-1394/38/2/4)

View the table of contents for this issue, or go to the journal homepage for more

Download details:

IP Address: 147.96.14.16

This content was downloaded on 29/05/2014 at $14: 28$

Please note that terms and conditions apply. 


\title{
On Babinet's principle and a diffraction-interferometric technique to determine the diameter of cylindrical wires
}

\author{
J. C. Martínez-Antón, I. Serroukh \\ and E. Bernabeu
}

\begin{abstract}
Laser diffraction is a robust and precise technique for measuring wire diameters. However, classical Fraunhofer diffraction formulas are inappropriate for determining the size of three-dimensional objects. Babinet's principle (from classical diffraction theory) allows the use of such formulas only for angles of diffraction tending to zero. In practice diffraction measurements require a finite angular range (about $10^{\circ}$ ) for good resolution. We show that use of the classical slit-diffraction formula for this extended angular interval introduces significant error. We develop a simple diffraction model valid for cylindrical shapes and a formula to obtain the diameter of cylindrical wires. To validate the diffraction formula we calibrate the diameter of the wires using an independent interferometric technique, which to our knowledge is original for this particular task. We have verified our diffraction formula to within about $0.2 \mu \mathrm{m}$ for various wire materials and diameters $(30 \mu \mathrm{m}$ to $300 \mu \mathrm{m})$ and a wide angular range $\left( \pm 25^{\circ}\right)$. Two different experimental set-ups were implemented to increase the reliability of the results. The calibration procedure revealed a systematic disagreement with Babinet's principle, in particular a systematic overestimation of the diameter by about $0.6 \mu \mathrm{m}$, almost independent of the material and the diameter itself. This is particularly relevant to metrological applications involving diffraction phenomena and based on classical diffraction theory.
\end{abstract}

\section{Introduction}

The original motivation of this work was to improve the diffraction model for measuring wire diameters. The classical theory of diffraction is based on the Huygens-Fresnel principle and the Fresnel-Kirchhoff representation of the diffracted wave as a surface integral over the unobstructed portion of the wave, assuming an infinitely flat screen or obstacle. By means of a Maggi transformation, this result can be transformed into a line integral over the edge of the obstacle. Thus a diffraction pattern may be reinterpreted in terms of a boundary diffraction wave generated on the boundaries of real objects [1]. One consequence is Babinet's principle which, in terms of our particular problem, means that a cylindrical wire should produce the same diffraction pattern as a slit (or a strip) of the same projected diameter. Rigorously, Babinet's principle should only be applied to three-dimensional objects for very small angles of diffraction [2]; the greater the angle of diffraction, the larger the error in associating a wire-diffraction pattern to a slit-diffraction pattern. The main objective of the current work is to derive and experimentally check a diffraction formula

J. C. Martínez-Antón, I. Serroukh and E. Bernabeu: Departamento de Optica, Facultad de Físicas, Universidad Complutense de Madrid, 28040 Madrid, Spain.

e-mail: fiopt12@emducms1.sis.ucm.es valid for cylindrical wires of any size. As a spin-off we have observed and quantified a "disagreement" with Babinet's principle which, if not considered explicitly, may lead to an error of over $1 \%$ for wires less than $60 \mu \mathrm{m}$ in diameter.

We propose a diffraction formula based on the simple model of the geometrical theory of diffraction (GTD) $[3,4]$. That is, we consider the diffraction pattern produced from interference between a diffracted "surface" ray and one reflected at the surface. Both types of ray can be justified in terms of the generalized Fermat's principle, which is used to calculate their optical paths and phases [5, 6].

Another approach to the problem is given by Lebrun et al. [7] by means of simulations using the Lorenz-Mie formulation. Although a direct comparison with the current paper is not possible, qualitative agreement is found in terms of the overestimation of the diameter when using classical Fraunhofer diffraction formulas $(\Delta d \approx 0.5 \mu \mathrm{m})$.

Most of the commercial laser diffraction instruments for determining wire diameter operate at angular ranges of $\pm 10^{\circ}$ or less. We show that this angular range is not enough to validate a diffraction formula from the diffraction pattern alone; calibration of the diameter is also required. We have developed an interferometric calibration procedure that allows simultaneous determinations of the diameter by interference and diffraction on the same portion of the 
wire. This is an accurate and reliable technique for calibrating a diffractometer as well as for investigating diffraction phenomena.

\section{Diffraction model}

As stated above, our analysis is based on the simple GTD model (see Figure 1), in which the diffraction pattern is determined by the interference of two lateral rays: one ray reflected (\#2) and the other "diffracted" (the surface ray, \#1). We can explain surface rays in terms of Fermat's principle, according to which points $\mathrm{A}_{1}$ and $\mathrm{C}_{1}$ are connected by a stationary trajectory (see Figure 1).

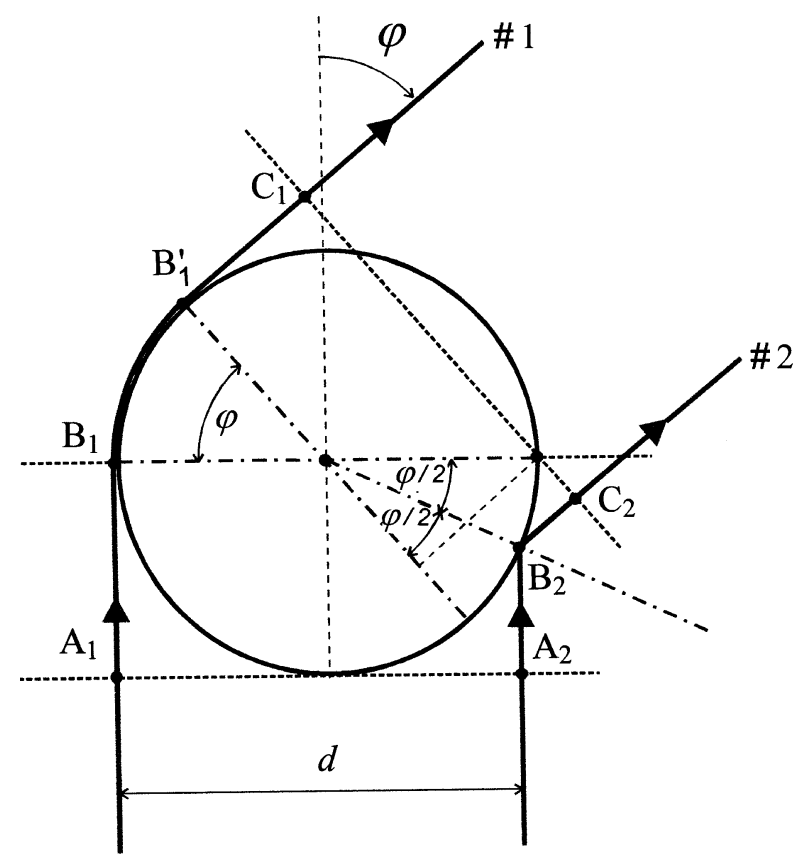

Figure 1. Proposed diffraction model based on the geometrical theory of diffraction (Keller, 1958, 1962 [3]). The incident beam defines the reference for the observation angle $\varphi$.

We are interested only in the periodic oscillations of the diffraction pattern (classically taken as the localization of the maxima and minima) which are a more robust source of information than only the radiation intensity. For this purpose the GTD is appropriate. Nevertheless, in the development of the formulation we will appreciate the need to define the interference extremes in terms of the envelopes of the diffraction pattern instead of referring to the maxima or the minima. In other words, the analytically useful features are the envelope extremes. We define these as the points where the diffraction pattern touches and is tangent to its envelopes. Figure 2 illustrates the difference between extremes and envelope extremes; this distinction is crucial for high-accuracy measurements.

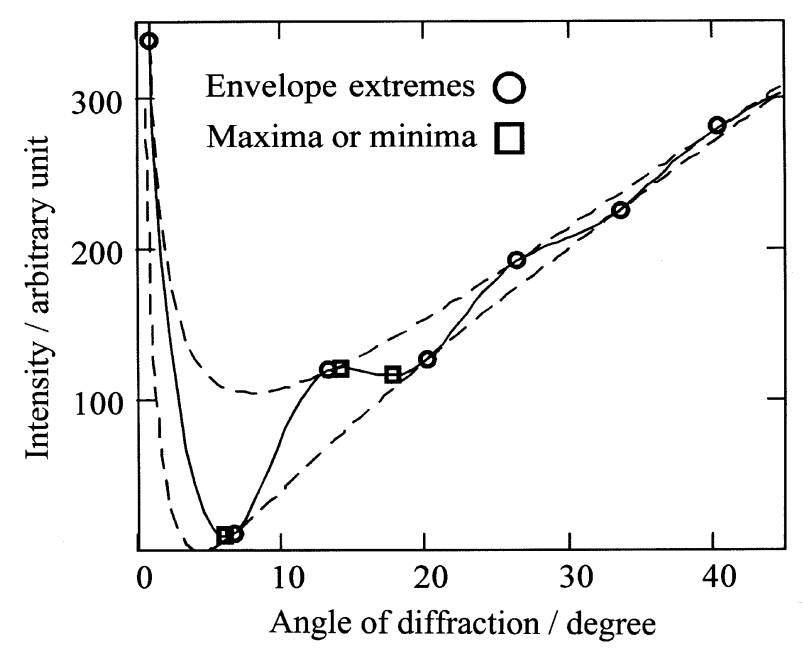

Figure 2. Difference between extremes (maxima or minima in squares) and envelope extremes (circles). The oscillating curve simulating a diffraction pattern is shown as a solid line and its upper and lower envelopes by dashed lines. The diffraction pattern is calculated with (4) and its envelopes with (6), taking appropriate $I_{1}(\varphi)$ and $I_{2}(\varphi)$.

In order to calculate the field-amplitude at the detector, situated at the angle of diffraction $\varphi$, we need to know the optical paths of the two rays. First we decompose the surface ray (\#1) into three distinct steps: the linear segment $A_{1} B_{1}$, the arc of circumference $\mathrm{B}_{1} \mathrm{~B}_{1}^{\prime}$ (the circumference is the wire profile), and the final linear segment $B_{1}^{\prime} C_{1}$. The total optical path of the surface ray may be written

$$
S_{1} \equiv\left[A_{1} C_{1}\right]=\frac{d}{2}(1+\varphi+\sin \varphi)
$$

where we have assumed that the surface ray "travels" in air but at zero distance from the surface. It is interesting to note that, from a radiometric point of view, the ray's path should be considered as being very close to the surface but not at zero distance, and this idea is corroborated by our experimental results. Within the framework of the GTD, however, we assume the rays to be adimensional.

Similarly, the optical path of the reflected ray (\#2) is

$$
\begin{aligned}
& S_{2} \equiv\left[A_{2} C_{2}\right]= \\
& \frac{d}{2}(1-\sin (\varphi / 2))+\frac{d}{2}(\sin (\varphi)-\sin (\varphi / 2)) .
\end{aligned}
$$

The optical path difference between the two rays is thus

$$
\Delta S=S_{1}-S_{2}=d\left[\varphi-\frac{\varphi^{3}}{48}+O\left(\varphi^{3}\right)\right]
$$

where we have taken a series expansion to the third power. This approximation may be justified as follows. 
Our measurements on $30 \mu \mathrm{m}$ to $300 \mu \mathrm{m}$ wires have an observable diffraction pattern only within an interval of about $\pm 20^{\circ}$ and only when the surface is very smooth (rougher wires produce smaller observable intervals). For this maximum interval the third-order term in (3) represents a contribution of less than $0.3 \%$ of the linear term, so higher-order terms can be ignored.

The optical path difference $\Delta S$ allows us to calculate the interference in the diffraction pattern. The intensity distribution at the detector location may be described by a function of type

$$
\begin{aligned}
I(\varphi)=E E^{*}= & \mid E_{1} \exp \left(i \frac{2 \pi}{\lambda} S_{1}+\delta_{1}\right)+ \\
& \left.E_{2} \exp \left[i\left(\frac{2 \pi}{\lambda} S_{2}+\delta_{2}\right)\right]\right|^{2} \\
=I_{1}(\varphi)+ & I_{2}(\varphi)+2 \sqrt{I_{1}(\varphi) I_{2}(\varphi)} \times \\
& \cos \left[\frac{2 \pi}{\lambda}\left(S_{1}-S_{2}\right)+\left(\delta_{1}-\delta_{2}\right)\right],
\end{aligned}
$$

where $E$ is the total field at the detector and subscripts 1 and 2 indicate the contributions from each side of the cylindrical wire. Classically, the positions of the extremes for diffraction are obtained from the interference condition

$$
\begin{aligned}
& \frac{2 \pi}{\lambda}\left(S_{1}-S_{2}\right)-\delta_{2 \mathrm{D}}=m \pi, \\
& \text { with } \quad m=0, \pm 1, \pm 2, \ldots,
\end{aligned}
$$

where $\delta_{2 \mathrm{D}}$ is the phase term of the reflected ray. We assume that there is no special phase term in the surface ray apart from the optical path contribution.

It is important to bear in mind that (5) gives the location of the fringe maxima and minima only when $I_{1}(\varphi)$ and $I_{2}(\varphi)$ are constant with $\varphi$. In fact, (5) really represents the location of the envelope extremes, i.e. the points where the diffraction envelope touches its own envelopes tangentially.

Substituting (5) in (4) defines the envelopes of the diffraction pattern:

$$
I_{\mathrm{env}}(\varphi)=\left(\sqrt{I_{1}(\varphi)} \pm \sqrt{I_{2}(\varphi)}\right)^{2} .
$$

In the case of a typical diffraction pattern, the envelope extremes coincide approximately with the fringe maxima and minima only when the envelopes are quasi-flat. For the upper envelope extremes in the diffraction pattern of Figure 5, this corresponds roughly to the interval $165^{\circ}$ to $175^{\circ}$ for the angle of observation $(\pi-\varphi)$.

From (3) and (5) we obtain a diffraction formula to determine the diameter of a cylindrical wire from the envelope extremes:

$$
\begin{aligned}
& \frac{m \lambda}{2 d}=\left[\varphi-\frac{\varphi^{3}}{48}+O\left(\varphi^{3}\right)\right]-\delta_{2 \mathrm{D}} \frac{\lambda}{2 \pi d}, \\
& \text { with } m=0, \pm 1, \pm 2, \pm 3, \ldots,
\end{aligned}
$$

The envelope maxima correspond to the even values of $m(m= \pm 2, \pm 4, \ldots)$ and the envelope minima to the odd values $(m= \pm 1, \pm 3, \ldots)$.

On the other hand, for the limiting case of small angles $(\varphi=0)$ we may assume that $\delta_{2 \mathrm{D}}$, the phase term due to reflection, is $\pi$, independent of the surface or material. For real measurements (finite $\varphi$ ), however, we have to calculate $\delta_{2 \mathrm{D}}$ using Fresnel equations [8]. Figure 3 shows the phase angle of the reflected ray versus the angle of incidence for two different polarizations ( $p$, perpendicular to the wire axis; $s$, parallel to the wire axis) incident on a metallic surface. In diffraction, the reflected rays are near grazing incidence. From the figure we see that the reflection phase $\delta_{2 \mathrm{D}}$ may be approximated in this region by

$$
\delta_{2 \mathrm{D}}=C_{0}+C_{1} \varphi,
$$

where $C_{0}=\pi$ and $C_{1}$ depends on the polarization, the material, and its surface properties.

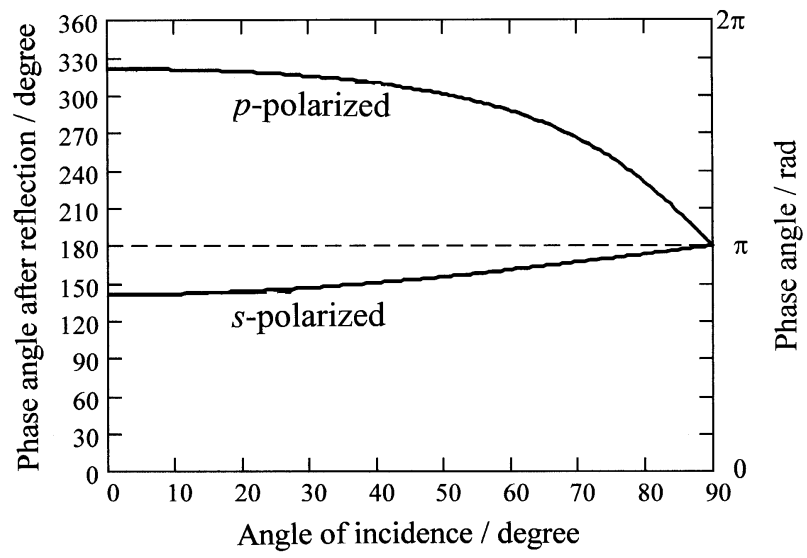

Figure 3. Typical angular distribution of the phase term (in degrees) arising from reflection at a metallic surface. The example shows gold ( $n=0.18, k=2.84$ ). The upper curve is for $p$-polarization (perpendicular to the wire axis) and the lower for $s$-polarization (parallel with the wire axis).

Using (8), (7) may also be expressed as

$$
\begin{aligned}
& \frac{m \lambda}{2 d}=\left[\left(1-C_{1} \frac{\lambda}{2 \pi d}\right) \varphi-\frac{\varphi^{3}}{48}+O\left(\varphi^{3}\right)\right], \\
& \text { with } m= \pm 1, \pm 2, \pm 3, \ldots
\end{aligned}
$$

If $C_{1}$ is known, (9) may be used to estimate the wire diameter from the diffraction pattern. For relatively thick diameters (i.e. $2 \pi d \gg \lambda C_{1}$ ), (7) may be rewritten in the more useful form

$$
\begin{aligned}
& \frac{m \lambda}{2 d_{\mathrm{D}}^{\prime}}=\left[\varphi-\frac{\varphi^{3}}{48}+O\left(\varphi^{3}\right)\right], \\
& \text { with } \quad m= \pm 1, \pm 2, \pm 3, \ldots,
\end{aligned}
$$

where

$$
d_{\mathrm{D}}^{\prime}=d+\mu_{\mathrm{D}}
$$


and $\mu_{\mathrm{D}}(\ll d)$ is

$$
\mu_{\mathrm{D}}=-\frac{C_{1} \lambda}{2 \pi} .
$$

\subsection{Babinet's principle and the symmetry of the problem}

Whatever the model, the derived formula must have the form:

$$
\frac{m \lambda}{2 d}=\left[a_{1} \varphi+a_{3} \varphi^{3}\right] .
$$

In this expression we have omitted the even terms simply by symmetry considerations, but in the analysis of the experimental data we use a polynomial with both odd and even powers. In practice there are various sources of asymmetry, such as surface roughness, departure from a cylindrical shape or simply certain asymmetries in the experimental set-up.

Babinet's principle is valid when the angle of observation $\varphi$ tends to zero, at which limit (13) should converge to the equivalent slit formula

$$
\frac{m \lambda}{2 d}=\sin \varphi
$$

For very small angles this takes the form

$$
\frac{m \lambda}{2 d}=\varphi,
$$

i.e. the coefficient $a_{1}$ in (13) must be $a_{1}=1$.

Classical diffraction assumes scalar fields. We see in (9) that inclusion of a polarization term causes a certain disagreement with Babinet's principle $\left(a_{1} \neq 1\right)$. This disagreement has been confirmed experimentally, but we show that the polarization effect cannot account for all of the deviation.

\subsection{Resolution limits}

We want to know how large the angular interval must be to obtain a certain resolution. In the current measurements the locations of the envelope extremes have been determined to a resolution $\rho(=\Delta m)$ of between one-tenth and one-fiftieth of a diffraction order, independent of the wire diameter. This is equivalent to a constant resolution in diameter of a fraction of a wavelength $\lambda$. Let us first assume that (15) is adequate to provide an estimate of the diameter resolution (the third-order contribution in (10) being less than $0.3 \%$ ). For a maximum angular interval of $\pm \varphi_{\max }$ we see that $m$ is $2 d \varphi_{\max } / \lambda$, which gives $\Delta d / d=\Delta m /\left(2 d \varphi_{\max } / \lambda\right)$. Thus the resolution is $\Delta d=\Delta m \lambda / 2 \varphi_{\max }$. For $\lambda=0.633 \mu \mathrm{m}, \Delta m=0.1$ and a typical $\pm 10^{\circ}$ interval, we find a resolution in diameter of approximately $0.2 \mu \mathrm{m}$ (independent of the wire diameter or the number of fringes). This resolution seems to be satisfactory but it is slightly above the contribution of the third-order term in the proposed formula (10) for wires of diameter less than $350 \mu \mathrm{m}$ and a diffraction measurement alone is therefore not enough to verify the third-order term. We must resort to a calibration procedure to measure the true diameter and to provide reliable polynomial coefficients in (13). The calibration procedure is presented in Section 3.

\section{Interferometric calibration}

Different methods of measuring the diameter of a wire may be found in the literature [4-12]. Calibration measurements are usually made by contact techniques [11]. Perhaps the main disadvantage of this type of calibration is the difficulty of guaranteeing that the area of wire as measured by the diffractometer is the same as that measured in the calibration. Normally, the sensing areas are different and slight inhomogeneities in diameter are unavoidable [11]. We propose a calibration method that overcomes this difficulty in a practical way and allows simultaneous calibration and measurement in a diffractometer. The idea is to use the interference of two coherent laser beams on the wire, as illustrated in Figure 4.

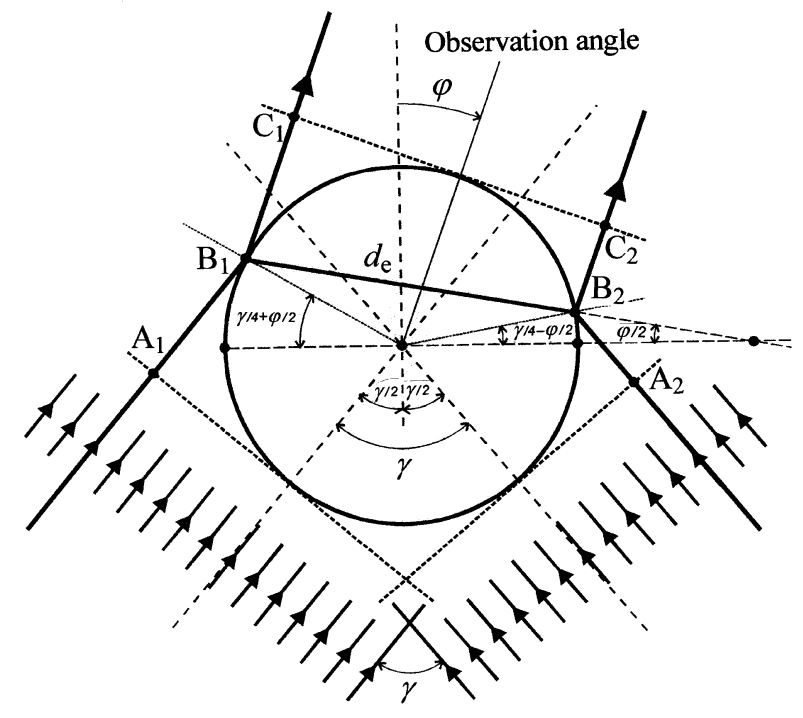

Figure 4. Two-beam interference on a wire. At observation angle $\varphi$ the appearance is of a double slit $\left(\mathrm{B}_{1} \mathrm{~B}_{2}\right)$ separated by a distance $d_{\mathrm{e}}$. This double slit is rotated by $\varphi / 2$ with respect to the angle of observation $\varphi$.

In the angular detection area we now have both diffracted light (in the sense of surface rays) and reflected light from the two beams. While the reflected component extends over the full $2 \pi$ angular range, in practice the diffraction pattern (which includes the surface rays) appears only within a small range (about $\pm 20^{\circ}$ ) (see Figure 5). Therefore, if the angle $\gamma$ between the incident beams is chosen appropriately, part of the 


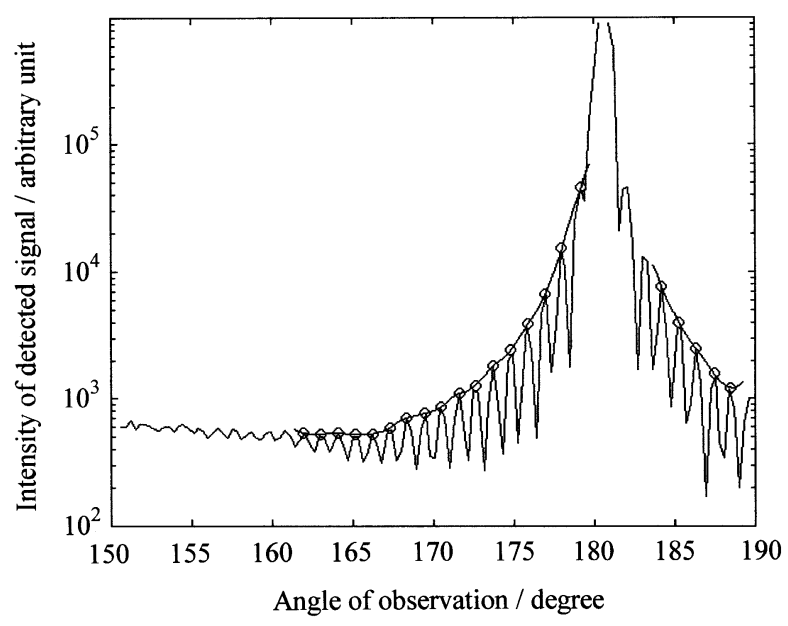

Figure 5. Diffraction pattern of a steel wire of $d=35 \mu \mathrm{m}$ obtained with the goniometric set-up. Note the wide dynamic range, not obtainable with a CCD. The upper envelope extremes of the diffraction pattern are partially traced.

angular field is occupied only by reflected light and in this region we have a pure interference pattern. Note that a relatively smooth, reflective wire surface is necessary to obtain such an interference pattern. In any case the diffraction model assumes a perfectly smooth surface.

Figure 4 shows that this interference problem is in practice equivalent to a Young's double slit, where the slits are the points $\mathrm{B}_{1}$ and $\mathrm{B}_{2}$, and the slit width is $d_{\mathrm{e}}$. The value of $d_{\mathrm{e}}$ is constant but, in contrast to Young's experiment, now the apparent double slit $\mathrm{B}_{1} \mathrm{~B}_{2}$ rotates by half the angle of observation $\varphi$. The extremes of the interference pattern may therefore be obtained from the following slightly modified Young's formula:

$$
\frac{m \lambda}{2 d_{\mathrm{e}}}=2 \sin \left(\frac{\varphi}{2}\right)
$$

where the effective diameter $d_{\mathrm{e}}$ is

$$
d_{\mathrm{e}}=d \cos \frac{\gamma}{4}
$$

To be free of the influence of diffraction in the interference pattern, $\gamma$ must be $40^{\circ}$ or more. For $\gamma=40^{\circ}$ we obtain $d_{\mathrm{e}}=0.985 d$, which means that the interference measurement "sees" almost the same diffraction diameter and area of the wire. This is ideal for calibration purposes.

Equation (16) does not include the effect of polarization, which we introduce now. The location of the envelope extremes in the interference pattern is given by

$$
\begin{aligned}
& \frac{2 \pi}{\lambda}\left[\left(S_{1}-S_{2}\right)+\left(\delta_{1 \mathrm{I}}-\delta_{2 \mathrm{I}}\right)\right]=m \pi, \\
& \text { with } \quad m=0, \pm 1, \pm 2, \ldots,
\end{aligned}
$$

where $S_{1}-S_{2}$ is the optical path difference between the two reflected rays in the $\varphi$ direction and $\delta_{1 \mathrm{I}}-\delta_{2 \mathrm{I}}$ is the difference in reflection phase. For the minima we obtain

$$
\begin{aligned}
& \frac{m \lambda}{2 d_{\mathrm{e}}}=\left[\varphi-\frac{\varphi^{3}}{24}+O\left(\varphi^{3}\right)\right]+\left(\delta_{1 \mathrm{I}}-\delta_{2 \mathrm{I}}\right) \frac{\lambda}{2 \pi d_{\mathrm{e}}}, \\
& \text { with } \quad m=0, \pm 1, \pm 3, \ldots .
\end{aligned}
$$

As in the previous section, the phase terms $\delta_{1 \mathrm{I}}$ and $\delta_{2 \mathrm{I}}$ can be modelled as

$$
\begin{aligned}
& \delta_{1 \mathrm{I}}=B_{0}-B_{1} \varphi, \\
& \delta_{2 \mathrm{I}}=B_{0}+B_{1} \varphi .
\end{aligned}
$$

From (19) and (20) we obtain for the minima

$$
\begin{aligned}
& \frac{m \lambda}{2 d_{\mathrm{I}}^{\prime}}=\left[\varphi-\frac{\varphi^{3}}{24}+O\left(\varphi^{3}\right)\right], \\
& \text { with } \quad m= \pm 0, \pm 1, \pm 3, \ldots,
\end{aligned}
$$

where

$$
d_{\mathrm{I}}^{\prime}=d \cos (\gamma / 4)+\mu_{\mathrm{I}}
$$

and $\mu_{\mathrm{I}}(\ll d)$ is

$$
\mu_{\mathrm{I}}=-\frac{B_{1} \lambda}{\pi} .
$$

It is interesting to note that $B_{1} \approx C_{1}$ (see Figure 3) so $\mu_{\mathrm{I}} \approx 2 \mu_{\mathrm{D}}$. In principle, $s$-polarization is chosen in order that $\mu_{\mathrm{I}}$ and $\mu_{\mathrm{D}}$ are negligible. From different simulations we obtain $\left|\mu_{\mathrm{I}}\right| \approx 2\left|\mu_{\mathrm{D}}\right|<0.1 \mu \mathrm{m}$ in $s$ polarization.

\section{Experimental set-ups}

We used two different experimental set-ups that allow simultaneous diffraction and interference measurements. Figure 6 shows part of the first set-up. The interferometric measurement is made between the two beams. Each laser beam in turn is also used to measure a diffraction pattern with a goniometric system [13] centred in the wire. In this way small asymmetries may be revealed, because each beam "sees" a different projection of the wire. The comparison between these three diameters is strongly correlated.

The goniometric equipment [13] has an angular resolution of $0.002^{\circ}$. The uncertainty of the angular positioning is estimated to be about $0.05^{\circ}$ (for angles over $50^{\circ}$ ), which limits the accuracy of the diameter determination to a relative uncertainty of 1 part in $10^{3}$ $\left(\Delta d_{\max } \approx 0.3 \mu \mathrm{m}\right)$. The size of the beams at the wire location is of the order of $2 \mathrm{~mm}$. In terms of diffraction, this is insignificant compared with the size of the wire. 


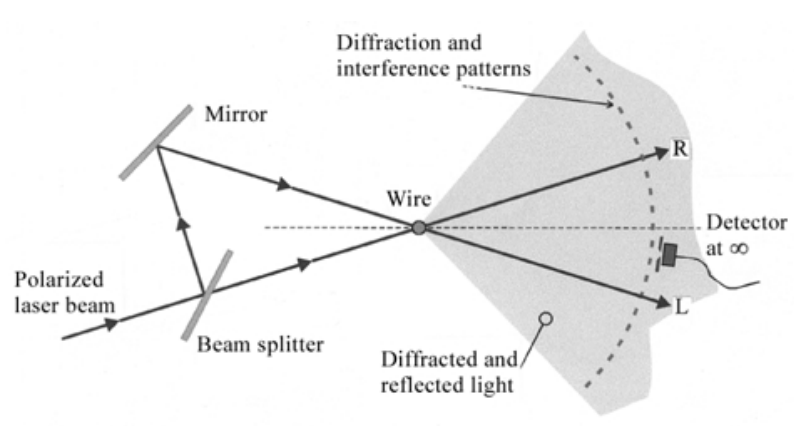

Figure 6. Scheme of the first experimental set-up. The reference to the observation angle $\varphi$ is the direction set by the bisectrix of the two laser beams. The detector is positioned at the focal plane of a spherical mirror (not shown) to simulate the infinity position. The rotation axis of the detector is coincident with the wire axis. The wire is perpendicular to the plane defined by the beams.

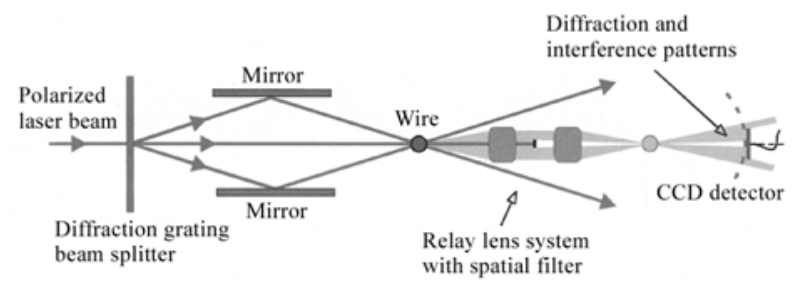

Figure 7. Scheme of the experimental set-up based on a CCD arrangement. The field-of-view of the CCD measures the diffraction pattern of the central beam and the interference pattern given by the two lateral beams. The alternate occlusion of the central beam and the lateral beams allows the interference and diffraction patterns to be detected separately. A relay system is introduced to filter the zero diffraction order to avoid saturation of the CCD.

In the first set-up the measurement procedure followed is: (a) measure the diffraction pattern given by the "left" beam (L) while keeping the "right" beam (R) off; (b) as (a) but with the "right" beam on and the "left" beam off; and (c) measuring the interference pattern in between, i.e. around the broken straight line in Figure 6.

Figure 7 shows the second experimental set-up. A diffraction grating is used as a beam splitter. The beams of order $-1,0$ and 1 cross at the wire. The three beams are in a common plane perpendicular to the wire. To avoid saturation of the detector by the direct incidence of the zero-order beam, a relay optical system is introduced after the wire with a zero-order spatial filter. The relay system consists of two eyepieces with a magnification of $1: 1$, with the exit pupils situated at conjugate points. The wire is situated at one exit pupil. This configuration helps reduce aberrations, particularly distortion, although complete removal of aberrations is not critical for this type of measurement. The most important issue is to align the system and to make it symmetrical. This was possible to within about 2 arcmin (roughly $6 \times 10^{-4} \mathrm{rad}$ ). The fringe pattern is detected on a $2 / 3$ format charge-coupled-device (CCD) detector $(512 \times 512$ pixels $)$ situated sufficiently far from the imaged wire for the Fraunhofer approximation to apply. The field-of-view of the CCD was always within a span of $\pm 3^{\circ}$ from the imaged wire.

The measurement procedure is as follows. We occlude the zero-order beam (before the wire) and register the interference pattern on the CCD. We then free the central beam and occlude the two lateral beams (orders 1 and -1 ). In this way we obtain the diffraction pattern of the wire. Between these two steps no mechanical change is made to the set-up apart from blocking or unblocking the laser beams. The time gap between measurements is short enough to guarantee that any possible drift (mechanical, thermal or other) is negligible. This is in contrast to the instrument presented previously [13], with which each measurement took at least $10 \mathrm{~min}$ to complete. With that instrument we could only assure a fringe drift (in the interference pattern) of less than 0.1 fringe over the whole measurement, which led to a limiting absolute uncertainty of about $0.2 \mu \mathrm{m}$ in the diameter of the wire. With the CCD we improve the resolution of the diffraction-interference comparison to below $0.1 \mu \mathrm{m}$.

\section{Analytical procedures}

The angle of observation can only be obtained accurately using the goniometric set-up. To fit the envelope-extremes data to the theoretical model (in both the diffraction and the interference patterns) we used the generic formula (13), but with the inclusion of even terms (to allow for asymmetries):

$$
\frac{m \lambda}{2 d^{\prime}}=\left[a_{0}+a_{1} \varphi+a_{2} \varphi^{2}+a_{3} \varphi^{3}\right]
$$

According to our diffraction model, we expect the coefficients to be $a_{1}=1, a_{0}=a_{2}=0$ and $a_{3}=-1 / 48$.

More specifically, we make a fit to the associated equation

$$
m=\left[c_{0}+c_{1} \varphi+c_{2} \varphi^{2}+c_{3} \varphi^{3}\right]
$$

where $c_{i}=a_{i} 2 d^{\prime} / \lambda$. Using the experimental $c_{1}$ value and setting $a_{1}=1$ (according to Babinet's principle) we obtain $d^{\prime}$. At least in $s$-polarization we expect a negligible difference $(0.05 \mu \mathrm{m}$ at most) between the diffraction diameter and the interference calibrated diameter.

A different procedure is followed for the CCD set-up, as the CCD only allows the measurement of small angular intervals. In this case we can model the locations of the diffraction and interference minima without including the second- and third-order terms in (24):

$$
\frac{m \lambda}{2 d^{\prime}}=\left[a_{0}+a_{1} \varphi\right], \quad \text { with } \quad m=0, \pm 1, \pm 3, \ldots
$$


Neglecting the second- and third-order terms in (26) leads to an estimated error of less than $2 \times 10^{-4} d$ for a total field-of-view of about $6^{\circ}$ (for both diffraction and interference measurements), that is, less than $0.1 \mu \mathrm{m}$ error in diameter for $d \ll 400 \mu \mathrm{m}$. The coefficient $a_{1}$ is expected to be unity for both diffraction and interference, according to (10) and (21), respectively. The parameter $d^{\prime}$ is the effective diameter in diffraction, $d_{\mathrm{D}}^{\prime}$, or interference, $d_{\mathrm{I}}^{\prime}[(11)$ and (22)]. Equation (26) includes a zero-order term to account for small asymmetries.

From (26) and two simultaneous measurements of diffraction and interference we can obtain the ratio $d_{\mathrm{D}}^{\prime} / d_{\mathrm{I}}^{\prime}$ directly as follows. The output experimental variables are the counts (intensity) and the pixel numbers of the CCD. Ideally we should know the function that links the pixel positions with the angle $\varphi$, but in practice we know this function only approximately and it is better to perform the comparison between diffraction and interference using the pixel numbers directly. The relation between the angle $\varphi$ and the pixel number $x$ may be modelled by

$$
\begin{aligned}
& \varphi=\left[b_{0}+b_{1} x+b_{2} x^{2}+b_{3} x^{3}+\ldots\right], \\
& \text { with } \quad x=0, \pm 1, \pm 2, \pm 3, \ldots,
\end{aligned}
$$

where $b_{i}$ are coefficients that depend on the instrument configuration and are independent of whether we are performing a diffraction or an interference measurement. From (27) and substituting $a_{1}=1$ in (26),

$$
m=\frac{2 d^{\prime}}{\lambda}\left[a_{0}+b_{0}+a_{1} b_{1} x+a_{1} b_{2} x^{2}+a_{1} b_{3} x^{3}\right] .
$$

Alternatively,

$$
m=\left[c_{0}+c_{1} x+c_{2} x^{2}+c_{3} x^{3}+\ldots\right],
$$

with $c_{0}=\left(a_{0}+b_{0}\right) 2 d^{\prime} / \lambda, c_{1}=b_{1} 2 d^{\prime} / \lambda, c_{2}=b_{2} 2 d^{\prime} / \lambda$ and $c_{3}=b_{3} 2 d^{\prime} / \lambda$. The coefficients $c_{i}$ are directly obtainable from a fit to the CCD data (in diffraction or interference). Our goal now is to compare $d_{\mathrm{D}}^{\prime}$ and $d_{\mathrm{I}}^{\prime}$. We obtain the ratio between them from the ratio between the $c_{i}$ coefficients of (29), defined for diffraction and interference, i.e.

$$
\frac{d_{\mathrm{D}}^{\prime}}{d_{\mathrm{I}}^{\prime}}=\left[\frac{c_{1 \mathrm{D}}}{c_{1 \mathrm{I}}}=\ldots=\frac{c_{3 \mathrm{D}}}{c_{3 \mathrm{I}}}\right]
$$

We choose the first ratio $\left(c_{1 \mathrm{D}} / c_{1 \mathrm{I}}\right)$ in order to determine $d_{\mathrm{D}}^{\prime} / d_{\mathrm{I}}^{\prime}$ more accurately, because the measurements estimate the $c_{1}$ coefficients better.

From (11) and (22) we may write

$$
\frac{d_{\mathrm{D}}^{\prime}}{d_{\mathrm{I}}^{\prime}} \cos (\gamma / 4)=\frac{d_{\mathrm{D}}}{d_{\mathrm{I}}}+\frac{\mu_{\mathrm{DI}}}{d_{\mathrm{I}}},
$$

where $\mu_{\mathrm{DI}}$ is very approximately $\mu_{\mathrm{D}}-\mu_{\mathrm{I}}$ and we have used the fact that $\mu_{\mathrm{I}} \approx 2 \mu_{\mathrm{D}} \ll d$. In theory $d_{\mathrm{I}}=d_{\mathrm{D}}$ but, in practice, we have found significant systematic differences. This difference may be expressed in the following way:

$$
\frac{d_{\mathrm{D}}}{d_{\mathrm{I}}}=1+\frac{\Delta d_{\mathrm{DI}}}{d_{\mathrm{I}}},
$$

where

$$
\Delta d_{\mathrm{DI}}=d_{\mathrm{D}}-d_{\mathrm{I}},
$$

which accounts for an extra difference with respect to Babinet's principle. Finally, from (30), (31) and (32) we obtain

$$
\frac{\Delta d_{\mathrm{DI}}+\mu_{\mathrm{DI}}}{d_{\mathrm{I}}}=\cos \left(\frac{\gamma}{4}\right) \frac{c_{1 \mathrm{D}}}{c_{1 \mathrm{I}}}-1
$$

We use this formula to estimate $\Delta d_{\mathrm{DI}}^{\prime}=\Delta d_{\mathrm{DI}}+\mu_{\mathrm{DI}}$.

\section{Experimental results}

From the goniometric set-up we typically obtain diffraction patterns, as shown in Figure 5, and interference patterns, as shown in Figure 8. We have measured different metallic wires (gold, silver and steel) and the results, including the diameters $d^{\prime}$, obtained as explained above, are given in Tables 1 to 4 . Figure 9 represents a typical fit, compared with the classical slit-diffraction formula. It is clear that the classical formula holds only within a narrow angular range, and fails for angles greater than a few degrees. Weighting the most reliable measurements (gold and silver), the averaged $a_{i}$ coefficients among the different wires and measurements are $a_{2}=0.002 \pm 0.004$ and $a_{3}=-0.01 \pm 0.03$ (where the number following the symbol \pm is the numerical value of the standard deviation and not a confidence interval; the fitting accuracy is typically one order of magnitude less). These terms always contribute less than $0.25 \%$ of the linear term (for an angular measuring range of less than $\pm 25^{\circ}$ ). This leads to the important conclusion that the developed formula (13) is correct except for the small asymmetries (surface roughness, departure from cylindrical shape and misalignments) which may make a significant contribution to the $a_{2}$ and $a_{3}$ terms, masking the theoretical terms of (13) $\left(a_{2}=0\right.$ and $a_{3}=-1 / 48=-0.0208$ ).

Tables 1 to 4 also give the difference between the diameters measured with $p$ - and $s$-polarizations: $\Delta d_{p s}^{\prime}$. For the three materials involved we estimate that $\Delta d_{p s}^{\prime}$ is between $0.6 \mu \mathrm{m}$ and $0.7 \mu \mathrm{m}$. We used the following refractive indices: $\tilde{n}$ (gold) $=0.18-2.84$, $\tilde{n}($ silver $)=0.27-3.34 \mathrm{i}, \tilde{n}$ (steel $)=2.8-3.34 \mathrm{i}$. From the tables we find $\Delta d_{p s}^{\prime}$ values typically from $0.4 \mu \mathrm{m}$ to $0.7 \mu \mathrm{m}$, which represents fairly good agreement with 


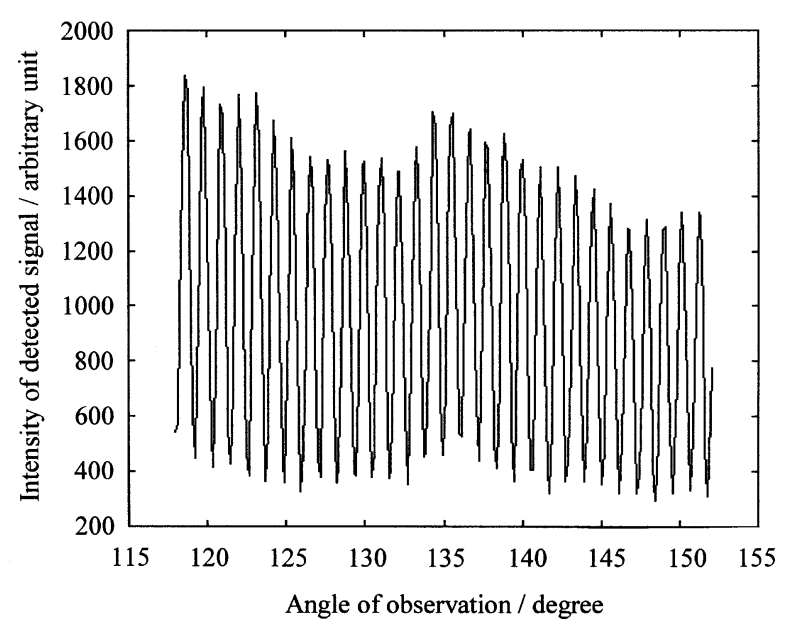

Figure 8. Typical interference pattern with the goniometric set-up. The plotted angle of observation is linearly related to the true observation angle $\varphi$ used in the formulas.

Table 1. Goniometric set-up: diffraction and interference results for a silver wire of nominal diameter $125 \mu \mathrm{m}$.

Zones 1 and 2 indicate different areas of the wire measured. See text for details.

\begin{tabular}{|c|c|c|c|c|}
\hline Silver & & Interference & Diffraction L & Diffraction $\mathrm{R}$ \\
\hline $\begin{array}{l}\text { Nominal } \\
125 \mu \mathrm{m} \\
\text { hp0 series }\end{array}$ & $\begin{array}{l}d_{p}^{\prime} / \mu \mathrm{m} \\
d_{s}^{\prime} / \mu \mathrm{m} \\
\Delta d_{p s}^{\prime} / \mu \mathrm{m} \\
\Delta d_{\mathrm{DI} s} / \mu \mathrm{m}=0\end{array}$ & $\begin{array}{l}129.17 \\
128.6 \\
0.57 \pm 0.2 \\
0.35 \pm 0.2\end{array}$ & $\begin{array}{l}129.3 \\
128.74 \\
0.56 \pm 0.2 \\
\Delta d_{\mathrm{DI}_{p}}^{\prime} / \mu \mathrm{m}=\end{array}$ & $\begin{aligned} & 129.95 \\
& 129.16 \\
& 0.79 \pm 0.1 \\
&= 0.45 \pm 0.2\end{aligned}$ \\
\hline $\begin{array}{l}\text { Nominal } \\
125 \mu \mathrm{m} \\
\mathrm{hp} 3 \text { series }\end{array}$ & $\begin{array}{l}\left.d_{p}^{\prime} / \mu \mathrm{m} \text { (zone } 1\right) \\
\left.d_{s}^{\prime} / \mu \mathrm{m} \text { (zone } 2\right) \\
\Delta d_{p s}^{\prime} / \mu \mathrm{m} \\
\Delta d_{\mathrm{DI} s}^{\prime} / \mu \mathrm{m}=0\end{array}$ & $\begin{array}{l}128.67 \\
128.6 \\
- \\
.36 \pm 0.2\end{array}$ & $\begin{array}{l}128.85 \\
128.60 \\
0.6 \pm 0.1 \\
\Delta d_{\mathrm{DI} p}^{\prime} / \mu \mathrm{m}=\end{array}$ & $\begin{array}{l}129.4 \\
129.45 \\
- \\
=0.43 \pm 0.2\end{array}$ \\
\hline
\end{tabular}

Table 2. Goniometric set-up: diffraction and interference results for a gold wire of nominal diameter $125 \mu \mathrm{m}$.

\begin{tabular}{lllll}
\hline Gold & \multicolumn{5}{c}{ Interference } & Diffraction L Diffraction R \\
\hline Nominal & \multicolumn{4}{c}{} \\
$125 \mu \mathrm{m}$ & $d_{p}^{\prime} / \mu \mathrm{m}$ & 124.62 & 125.41 & 125.27 \\
ho5 series & $d_{s}^{\prime} / \mu \mathrm{m}$ & 124.12 & 124.88 & 124.91 \\
& $\Delta d_{p s}^{\prime} / \mu \mathrm{m}$ & $0.50 \pm 0.2$ & $0.53 \pm 0.1$ & $0.36 \pm 0.2$ \\
& $\Delta d_{\text {DI } s}^{\prime} / \mu \mathrm{m}=0.77 \pm 0.2$ & $\Delta d_{\mathrm{DI} p}^{\prime} / \mu \mathrm{m}=0.72 \pm 0.2$ \\
Nominal & \multicolumn{4}{c}{} \\
$125 \mu \mathrm{m}$ & $d_{p}^{\prime} / \mu \mathrm{m}$ & 124.51 & 125.30 & 125.22 \\
ho3 series & $d_{s}^{\prime} / \mu \mathrm{m}$ & 123.87 & 124.65 & 124.65 \\
& $\Delta d_{p s}^{\prime} / \mu \mathrm{m}$ & $0.64 \pm 0.1$ & $0.65 \pm 0.1$ & $0.57 \pm 0.1$ \\
& $\Delta d_{\mathrm{DI} s}^{\prime} / \mu \mathrm{m}=0.78 \pm 0.2$ & $\Delta d_{\mathrm{DI} p}^{\prime} / \mu \mathrm{m}=0.75 \pm 0.2$ \\
\hline
\end{tabular}

theory considering that the surface conditions, surface roughness, and presence of oxide layers (in steel), may have a considerable effect on the results. Xie et al. [12] give similar results for the $p / s$ differences.

The parameter $\Delta d_{\mathrm{DIs}}^{\prime}$ is the difference between the diameter measured by diffraction with respect to interference in $s$-polarization (similarly $\Delta d_{\mathrm{DI} p}^{\prime}$ in $p$ polarization). The diffraction diameter taken is the
Table 3. Goniometric set-up: diffraction and interference results for steel wires of nominal diameters $150 \mu \mathrm{m}$ and $300 \mu \mathrm{m}$. In both cases the surface is rougher than for the other wires.

\begin{tabular}{lllll}
\hline Steel (1) & \multicolumn{5}{c}{ Interference } & Diffraction L Diffraction R \\
\hline Nominal & & & \\
$150 \mu \mathrm{m}$ & $d_{p}^{\prime} / \mu \mathrm{m}$ & $148.1 \pm 0.2$ & 148.46 & 149.44 \\
ha6 series & $d_{s}^{\prime} / \mu \mathrm{m}$ & - & - & - \\
& $\Delta d_{p s}^{\prime} / \mu \mathrm{m}$ & - & - & - \\
& - & & $\Delta d_{\mathrm{DI} p}^{\prime} / \mu \mathrm{m}=0.62 \pm 0.3$ \\
Nominal & & & & \\
$300 \mu \mathrm{m}$ & $d_{p}^{\prime} / \mu \mathrm{m}$ & 302.38 & 302.96 & $302.08 ?$ \\
ha3 series & $d_{s}^{\prime} / \mu \mathrm{m}$ & 302.25 & 302.3 & 302.45 \\
& $\Delta d_{p s}^{\prime} / \mu \mathrm{m}$ & $0.13 \pm 0.3$ & $0.66 \pm 0.2$ & $-0.32 \pm 0.2$ \\
& $\Delta d_{\mathrm{DI} s}^{\prime} / \mu \mathrm{m}=0.13 \pm 0.3$ & $\Delta d_{\mathrm{DI} p}^{\prime} \mu \mathrm{m}=0.14 \pm 0.3$ \\
\hline
\end{tabular}

Table 4. Goniometric set-up: diffraction and interference results for a steel wire of nominal diameter $35 \mu \mathrm{m}$. This wire came from a different supplier to steel (1) and was smoother.

\begin{tabular}{lllll}
\hline Steel (2) & \multicolumn{5}{c}{ Interference } & Diffraction L Diffraction R \\
\hline Nominal & \multicolumn{4}{c}{} \\
$35 \mu \mathrm{m}$ & $d_{p}^{\prime} / \mu \mathrm{m}$ & 34.0 & 34.74 & 34.72 \\
ht3 series & $d_{s}^{\prime} / \mu \mathrm{m}$ & 33.58 & 34.2 & 34.23 \\
& $\Delta d_{p s}^{\prime} / \mu \mathrm{m}$ & $0.42 \pm 0.2$ & $0.44 \pm 0.1$ & $0.35 \pm 0.1$ \\
& $\Delta d_{\mathrm{DI} s}^{\prime} / \mu \mathrm{m}=0.73 \pm 0.2$ & $\Delta d_{\mathrm{DI} p}^{\prime} / \mu \mathrm{m}=0.63 \pm 0.2$ \\
Nominal & \multicolumn{4}{|c}{} \\
$35 \mu \mathrm{m}$ & $d_{p}^{\prime} / \mu \mathrm{m}$ & 34.1 & 34.36 & 34.8 \\
ha7 series & $d_{s}^{\prime} / \mu \mathrm{m}$ & 33.68 & 33.93 & 34.22 \\
& $\Delta d_{p s}^{\prime} / \mu \mathrm{m}$ & $0.42 \pm 0.2$ & $0.43 \pm 0.1$ & $0.58 \pm 0.1$ \\
& $\Delta d_{\mathrm{DI} s}^{\prime} / \mu \mathrm{m}=0.38 \pm 0.2$ & $\Delta d_{\mathrm{DI} p}^{\prime} / \mu \mathrm{m}=0.48 \pm 0.2$ \\
\hline
\end{tabular}

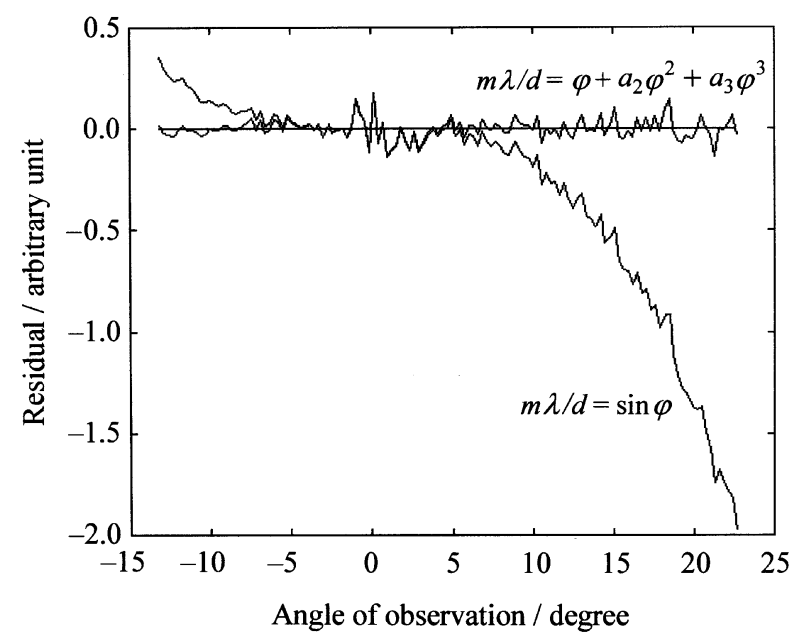

Figure 9. Residuals in diffraction order (assumed to be a continuous function of the angle) for a diffraction measurement of a smooth gold wire $(125 \mu \mathrm{m})$ and with the goniometric set-up [13]. Within an angular interval of about $\pm 5^{\circ}$ the error in $d$ is about $7 \%$; outside this range the classical slit-diffraction formula $m \lambda / d=\sin \varphi$ fails. Here $a_{2}=0.005$ and $a_{3}=-0.0025$. Both represent less than $0.2 \%$ of the linear term over the whole angular interval.

average between the left- and right-hand beam results. Note that there is often a significant difference between 
the two beams, indicating a degree of asymmetric deviation from the theoretical cylindrical shape. As expected there is also a significant difference between averaged diffraction and interference (see Table 5 for $\left.\Delta d_{\mathrm{DI}}^{\prime}\right)$ which cannot be explained by the polarization effect ( $\mu_{\mathrm{DI}}$ in the last column of Table 5).

Table 5. Systematic differences, extracted from Tables 1 to 4 , between diameters estimated by diffraction and by interferometry with $s$ - and $p$-polarizations. The last column is estimated from simulations.

\begin{tabular}{llll}
\hline $\begin{array}{l}\text { Goniometric } \\
\text { set-up }\end{array}$ & $\Delta d_{\mathrm{DI} s}^{\prime} / \mu \mathrm{m}$ & $\Delta d_{\mathrm{DI} p}^{\prime} / \mu \mathrm{m}$ & $\begin{array}{l}\text { Estimated } \\
\mu_{\mathrm{DI}} / \mu \mathrm{m}\end{array}$ \\
\hline Silver $(125 \mu \mathrm{m})$ & $0.35 \pm 0.2$ & $0.45 \pm 0.2$ & 0.109 in $p$-pol. \\
& $0.36 \pm 0.2$ & $0.43 \pm 0.2$ & 0.05 in $s$-pol. \\
Gold $(125 \mu \mathrm{m})$ & $0.77 \pm 0.2$ & $0.72 \pm 0.2$ & 0.001 in $p$-pol. \\
& $0.78 \pm 0.2$ & $0.75 \pm 0.2$ & 0.059 in $s$-pol. \\
Steel $(150 \mu \mathrm{m})$ & - & $0.62 \pm 0.3$ & \\
Steel $(300 \mu \mathrm{m})$ & $0.13 \pm 0.3$ & $0.14 \pm 0.3$ & 0.093 in $p$-pol. \\
Steel $(35 \mu \mathrm{m})$ & $0.73 \pm 0.2$ & $0.63 \pm 0.2$ & 0.031 in $s$-pol. \\
& $0.38 \pm 0.2$ & $0.48 \pm 0.2$ & \\
\hline
\end{tabular}

Table 6. Systematic differences (for $s$-polarization) between diameters estimated by diffraction (10) and interferometry (22), found with the CCD set-up. Different measurements mean a different orientation of the wire (by rotating it around its axis).

\begin{tabular}{llll}
\hline CCD set-up & $\Delta d_{\mathrm{DI} s}^{\prime} / \mu \mathrm{m}$ & $\left(<\Delta d_{\mathrm{DI} s}^{\prime}> \pm \sigma\right) / \mu \mathrm{m}$ & Estimated $\mu \mathrm{DI} / \mu \mathrm{m}$ \\
\hline Steel & $0.652 \pm 0.04$ & $0.67 \pm 0.1$ & 0.093 in $p$-pol. \\
$(400 \mu \mathrm{m})$ & $0.637 \pm 0.04$ & & 0.031 in $s$-pol. \\
& $0.838 \pm 0.04$ & \\
& $0.572 \pm 0.04$ & & \\
Gold & $0.696 \pm 0.05$ & $0.63 \pm 0.08$ & 0.001 in $p$-pol. \\
$(125 \mu \mathrm{m})$ & $0.544 \pm 0.05$ & & 0.059 in $s$-pol. \\
& $0.647 \pm 0.05$ & & \\
Silver & $0.738 \pm 0.05$ & $0.73 \pm 0.15$ & 0.109 in $p$-pol. \\
$(125 \mu \mathrm{m})$ & $0.880 \pm 0.05$ & & 0.05 in $s$-pol. \\
& $0.584 \pm 0.05$ & \\
\hline
\end{tabular}

We cannot measure $\mu_{\mathrm{D}}$ or $\mu_{\mathrm{I}}$. From simulations based on the known optical properties of various metals we find that the effect of polarization causes an offset in the estimated diameter, with values of $\mu_{\mathrm{D}}\left(\approx \mu_{\mathrm{I}}\right)$ from $0.5 \mu \mathrm{m}$ to $0.7 \mu \mathrm{m}$ for $p$-polarization and $-0.035 \mu \mathrm{m}$ to $-0.12 \mu \mathrm{m}$ for $s$-polarization.

The CCD configuration results (Table 6) lead to similar conclusions concerning $\Delta d_{\mathrm{DI}}^{\prime}$. Although the $\Delta d_{\mathrm{DI}}^{\prime}$ are only given for $s$-polarization they validate the goniometric results in the sense that classical diffraction leads to systematic overestimation of the diameter by about a wavelength (roughly $0.6 \mu \mathrm{m}$ ), almost independent of the material and the diameter (between $35 \mu \mathrm{m}$ and $400 \mu \mathrm{m}$ ).

It seems that the best diffraction formula to obtain the diameter of a smooth cylindrical metallic wire is $m \lambda /[2(d+\varepsilon)]=\varphi$, which must be used in $s$-polarization and within $\pm 10^{\circ}$ to obtain an uncertainty less than $0.1 \mu \mathrm{m}$. The parameter $\varepsilon$ is an "excess" diameter not predicted by the classical theory of diffraction. The theoretical effect of the polarization accounts for only a small fraction of $\varepsilon$ found experimentally. Fortunately, this empirical parameter $(\varepsilon)$ is approximately $0.6 \mu \mathrm{m}$ and almost independent of the wire diameter (between $35 \mu \mathrm{m}$ and $400 \mu \mathrm{m}$ ) and material. It is interesting that this value of $\varepsilon$ is of the same order as the wavelength used, in our case $\lambda=0.6328 \mu \mathrm{m}$. Any relation between $\varepsilon$ and $\lambda$ will have to be confirmed by additional experimental work.

\section{Conclusions}

We have developed and checked a simple diffraction formula for measuring the diameter of a cylindrical opaque wire, based on the geometrical theory of diffraction incorporating the effect of polarization. To validate such a formula we have developed an interferometric calibration technique. Two different experimental set-ups have been used, both of which have the advantage of analysing the same area of the wire simultaneously in diffraction and in interference. The goniometric set-up gives accurate estimations of the absolute diameter and the CCD set-up is very sensitive to small differences (diffraction/interference and $p$-/s-polarization). The diffraction model and the interferometric calibration give very similar results. We have verified (within the experimental uncertainty) the validity of the proposed diffraction theory over a wide angular range, and for different polarizations.

However, we have found that the wire diameter is systematically overestimated by both the classical slitdiffraction formula (for the limit of small angles) and the specifically developed diffraction formula. It seems that to obtain the diameter of a smooth cylindrical metallic wire a better formula is $m \lambda /[2(d+\varepsilon)]=\varphi$, which must be used in $s$-polarization and within $\pm 10^{\circ}$ to achieve an uncertainty less than $0.1 \mu \mathrm{m}$. The empirical parameter $\varepsilon$ is about $0.6 \mu \mathrm{m}$ and is almost independent of the wire diameter (between $35 \mu \mathrm{m}$ and $400 \mu \mathrm{m}$ ) and the material.

The existence of $\varepsilon$ contradicts Babinet's principle. However, from a rigorous point of view (from classical theory), a certain departure from Babinet's principle may be expected. Classical diffraction theory assumes that the field around the edge of any aperture is the incident field (Sommerfeld or Kirchhoff formulations). The present results clearly indicate that the electric field in the proximity of any real edge is not just the incident electric field as assumed in the classical approximation. Also, from a radiometric point of view, the rays should not be considered one-dimensional. Instead, they should better be considered in terms of finite "volume tubes" of energy, leading to an overestimation of the diameter that is almost independent of the material and the diameter itself. 
We believe that the results obtained are of particular interest in applied metrology. The overestimation of a wire diameter when using classical diffraction theory can give rise to a relative uncertainty of greater than $1 \%$ for wires thinner than $60 \mu \mathrm{m}$.

Acknowledgements. The authors gratefully acknowledge the support of the DEFCYL project (SMT 4-CT97-2184) from DG.12-EGAA of the European Community and the ABSODIAM project (TIC 961694-CE).

\section{References}

1. Nussenzveig H. M, Diffraction Effects in Semiclassical Scattering, Cambridge, Cambridge University Press, 1992, 17-20.

2. Greenler R. G., Hable J. W., Slane P. O., Am. J. Phys., 1990, 58(4), 330-331.

3. Keller J. B., J. Opt. Soc. Am., 1962, 52(2), 116-130.

4. Schmidt S., PTB-Mitteilungen, 1976, 86(4), 239-247.
5. Serroukh I., Martínez-Antón J. C., Bernabeu E., Proc. Conf. Wire Association International, Inc., 1999, 531-535.

6. Martínez-Antón J. C., Serroukh I., Bernabeu E., Proc. EOS/SPIE Symposium: Optical Measurements Systems for Industrial Inspection, 14-18 June 1999, Munich.

7. Lebrun D., Belaid S., Özkul C., Ren K. F., Gréhan G., Opt. Eng., 1996, 35(4), 946-950.

8. Azzam R. M. A., Bashara N. M., Ellipsometry and Polarized Light, Amsterdam, North-Holland, 1977.

9. Millard T. K., Herchenreder T. A., Wire J. Int., 1991, 24(12), 61-69.

10. Fardeau J. F., Wire J. Int., 1989, 22(1), 42-51.

11. Lipinski G., Priel M., Vailleau G. P., Metrologia, 1997, 34, 235-240.

12. Xie J., Qiu Y., Ming H., Li C., J. Appl. Phys., 1991, 69(10), 6899-6903.

13. Martínez-Antón J. C., Bernabeu E., Appl. Opt., 1994 , 33(25), 6059-6061.

Received on 7 February 2000 and in revised form on 31 August 2000. 